\title{
Study of the Current Limiting Device Based on Principle of Forced Current Zero
}

\author{
Qiang Li, Yu Tian, Weipeng Liang, Enyuan Dong, Jiyan Zou \\ Department of Electrical and Electronics Engineering \\ Dalian University of Technology \\ Dalian, 116024, China \\ E-mail: 380636145@qq.com
}

\begin{abstract}
Keywords-Current Limiting Device; Counter Pulse Circuit; Current Commutation; High Speed Switch (HSS); Control strategy
\end{abstract}

\begin{abstract}
With the development trend and rapid increase of the power grid capacity, short-circuit current level is increasing rapidly. How to make sure the steady and reliable operation of the power system is a big challenge. So it is of great importance to limit the short-circuit current effectively. A new kind of current limiting device based on current commutation is proposed in this paper, it consists of a high speed switch[1], counter pulse circuit and a limiting resistor. The function of a high speed switch is to open the fault current fast. The high speed switch can open the current at the forced current zero after a superposition of the counter pulse circuit. Then the current is commuted to a limiting resistor, the current limiting process is completed. According to the short-circuit current phase detection, the sequences coordination of the high speed switch and the counter pulse circuit, and the phase rule of three-phase short-circuit current, the control strategy at any phase of the three-phase short-circuit current is presented here. A circuit simulation is established by the Simulink tool box of Matlab. A lower current limiting experimental prototype is designed.
\end{abstract}

\section{Introduction}

At present, high-voltage switches of different parameters in different voltage levels are used as the primary actuator in delay protection of the power system in our country. When different types of short-circuit fault in the power system occur, the central control unit sends out the control signal to open the high-voltage switches, separating the fault component in a system from the normal section. However, there are two main problems in today's electric power system: First, due to the mechanical characteristics of circuit breaker itself, the time of breaking short-circuit current is long, from the occur of short circuit faults to the breaker opening to full stroke, it need 2-3 power cycle, meanwhile, fault current is far beyond the normal working current, therefore, the stability of the power system is impacted, electric equipment may be damaged, the system cannot guarantee the quality of electric energy. Second, along with the power dense degree increasing, the fault current level will continue to increase, but the circuit breaker itself is limited. The interrupting capacity of circuit breakers in power system is usually at the level of 63KA, it is with some technical problems to improve the voltage levels of circuit breakers. And replacing a lot of the existing circuit breakers in power system is not realistic. At present, methods for limiting the fault current is partition running. However, this method reduces the connection of the system. It is not conducive to the stability and reliability of the system.

The damage of short-circuit faults mainly depends on the volume of short-circuit current, thus, efficiently and quickly limiting the short-circuit current can solve the main problems listed above[2]. The current limiter proposed in this paper based on the principle of current commutation can quickly limit the short-circuit current. 


\section{Design of fault current limiter based on the current commutation principle}

For the above context, the fault current limiter we studied based on current commutation can avoid the impact of short-circuit peak current. We also give a discussion of control strategy of the whole cycle. Compared to the fault current limiter based on current commutation at power frequency zero[3,4], its performance improves significantly. This current limiter creates a rapid forced current zero after a superposition of the counter pulse circuit, and then quickly completes the current limiting process. It prevents the power system from larger impacts.

The principle of this current limiter:

Fig.1 shows a schematic diagram of the current limiter based on principle of current commutation. It consists of a high speed switch, a limiting resistor and a set of counter pulse circuit in the opposite direction (each counter pulse circuit is composed of a capacitor, an inductance, and a thyristor.AC short-circuit fault can occur in the positive half cycle or the negative half cycle, therefore, we build two counter pulse circuit). In addition, the most obvious feature of the current limiter is precise control. The fault current volume and phase detection is conducted through the current detection system. The logical analysis of fault current volume and phase is conducted through the central control unit. After the logic judgment, the central unit outputs the relevant control signals which control the corresponding counter pulse circuit and high speed switch, make the counter pulse circuit and high speed switch complete the current limiting process in cooperation. The high speed switch and counter pulse circuit need to be charged in advance, so the current limiter also includes some charging circuit, etc.

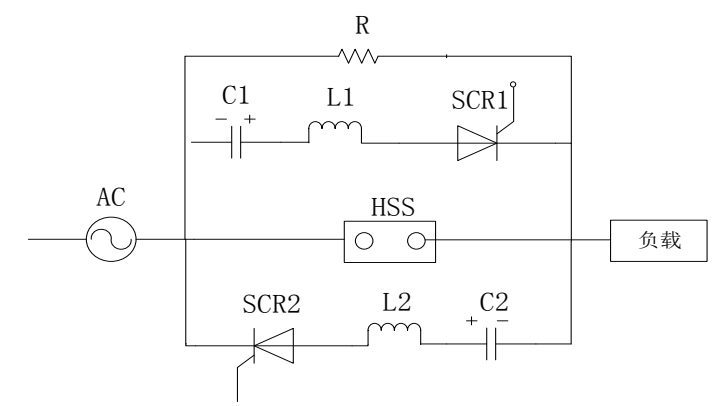

Figure 1. Schematic diagram of the current limiter

When there is no failure, the switch is closed, current flows through the switch. Since the current is in the normal range, the detection and control system does not issue a command, the entire current limiting device has no effects on the normal system.

When there are different types of short-circuit fault, line current increases sharply. When the short-circuit current is larger than a preset value, the central control unit issues a command to open the switch after the logic analysis. The high speed switch reaches full stroke position after 1.5ms. AC short-circuit current is not necessarily at zero point at this moment and the short-circuit current is not necessarily extinguished. So at this moment, the central unit sends a corresponding control signal to the corresponding counter pulse circuit or not sends this signal according to previous current detection and logic analysis. And then the electric arc is extinguished at the forced current zero. At the mean time, the fault current charges the capacitor from the opposite direction, SCR is also closed, and then the fault current is commuted to a limiting impedance branch. The current limiting process is completed.

\section{Discussion of control strategy}

When detecting fault current, the current limiter, researched in this project, will send control signals which are connected simultaneously by three-phase quick vacuum switch. After that, the main control problem has been converted to that the control unit sends out counter pulse drive circuit signal a, drive signal b, or doesn't send out counter pulse drive circuit signal according to the phase of fault current. As shown in figure 2, the control unit will send out drive signal a, when its 
arc point lies in positive half cycle, which corresponds to area a in the figure. The control unit will send out drive signal b, when its arc point lies in negative half cycle, which corresponds to area $b$ in the figure. When it doesn't send out drive signals, it indicates that the time quick switch reaches full stroke, fault current lies around zero point. Then natural arc can be extinguished through frequency zero point, so counter pulse circuit is not necessary. As shown in figure area c. The current limiter at this time is equal to the frequency zero current limiter. Taking phase $\mathrm{A}$ as an example, in one industrial frequency cycle $(20 \mathrm{~ms})$, when fault current is detected and the phase is judged, the sequence of control signals which are sent out within the whole cycle is shown in figure 2 . The cause for $1.5 \mathrm{~ms}$ lags is that when the system detects the fault current, the quick switch needs $1.5 \mathrm{~ms}$ to complete the operation from receiving control signal to reaching full stroke position.

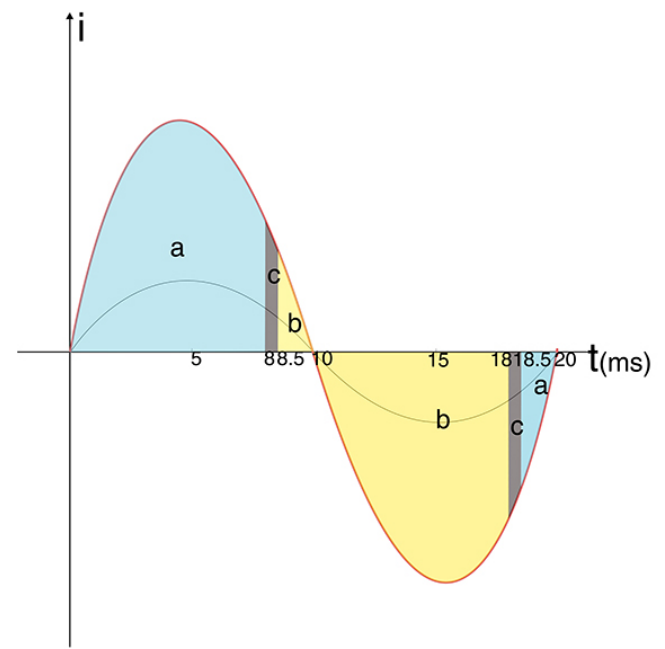

Figure 2. $\quad$ The control strategy of phase A

The three-phase alternating current has the regularity, namely sequential difference of phase A, phase $B$, and phase $C$ is $120^{\circ}$.In above figure, the fault current of phase $B$ and phase $C$ are also marked. As shown in figure 3, according to control strategy of counter pulse circuit of phase A and phase angle difference of phase $\mathrm{A}$, phase $\mathrm{B}$ and phase $\mathrm{C}$, the control signals of counter pulse circuit of phase $\mathrm{B}$ and phase $\mathrm{C}$ at the same time can be confirmed.

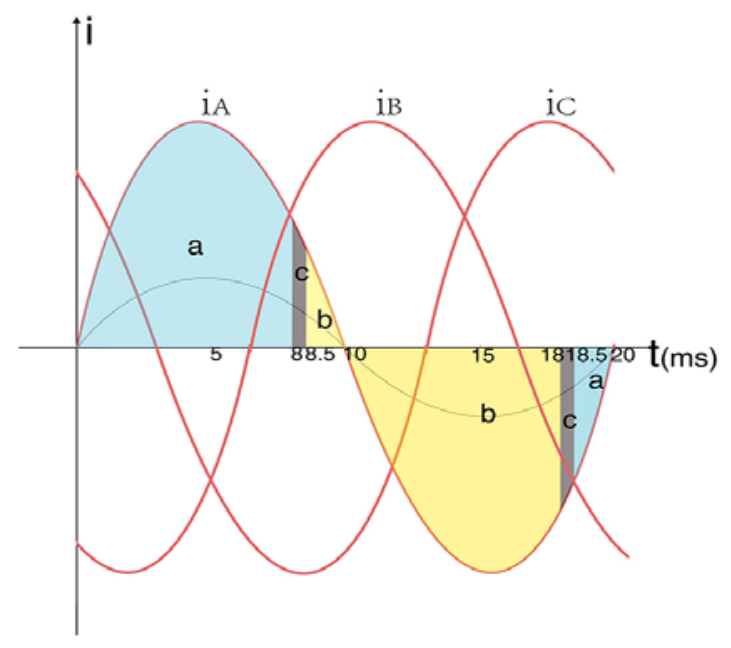

Figure 3. Corresponding diagram of three-phase fault current

\section{Simulations and experiment}

Building of simulation model

On the basis of circuit structure parameters of current limiter researched in this project, we make a simulation modeling study on single phase current limiting process by using Simulink tool box of Matlab. The simulation circuit is shown in figure 4. The fault current is produced by AC power of 
major loop and little value resistor $\mathrm{R}$. The aim is to simulate the actual system short-circuit process. In order to make circuit work in inductive mode, the inductance $\mathrm{L}$ is in series. Four branches of current limiter respectively are: A set of counter pulse circuit with opposite direction which are composed of C1, L1, GTO1 and C2, L2, GTO2; The breaker branch which high speed switch locates in; The current limiting branch which current-limiting impedance locates in. The simulative parameters of all elements are as following: frequency of AC power is of $50 \mathrm{HZ}$, peak voltage is of $10 \mathrm{KV}$, line inductance $\mathrm{L}$ is of $100 \mu \mathrm{H}$, and line impedance $\mathrm{R}$ is of $1 \Omega$. So the peak value of simulative fault current may be up to $10 \mathrm{KA} . \mathrm{C} 1=\mathrm{C} 2=110 \mu \mathrm{F}, \mathrm{L} 1=\mathrm{L} 2=35 \mu \mathrm{H}$, the initial voltage value of pulse capacitor is of $10 \mathrm{KV}$.

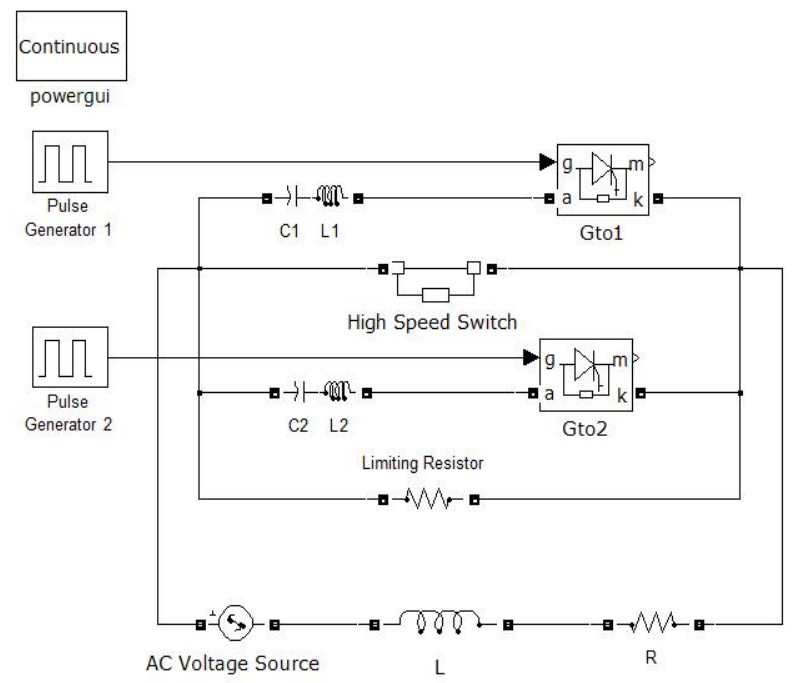

Simulation results and relevant analysis

Figure $4 . \quad$ Schematic diagram of simulation

The short-circuit simulation is at the level of $10 \mathrm{kV}$ voltage: The maximum peak current of shortcircuit is about 10kA. Short-circuit current's maximum peak is of $10 \mathrm{kA}$ which can be simulated through adjusting the voltage and impedance of main circuit. The system starts to simulate short circuit state, the time difference of the time that turning on the quick switch and the time that accessing counter pulse circuit is set to $1.5 \mathrm{~ms}$. The whole system coordinates to complete the current-limiting process. The simulation results of current-limiting process are shown in figure 5. From figure 5, waveform changes of different branches during current limiting process can be seen:

Before current limiting operation, the current, flows through main circuit and quick switch, is the same, no operation for counter pulse circuit, no current flows through current limiting resistance, and there is $10 \mathrm{KV}$ voltage precharged in pulse capacitor.

During the limiting process the short current is obviously limited, the fault current is only 5.5KA after current limiting process, which is about 55\% of original fault current. Because of the superposition of counter pulse current, the arc is extinguished at the forced current zero. Counter pulse circuit sends out a high frequency pulse current which exceeds 10KA. The limited fault current passes through the current-limiting resistance. The pulse capacitor is filled with a high reverse voltage.

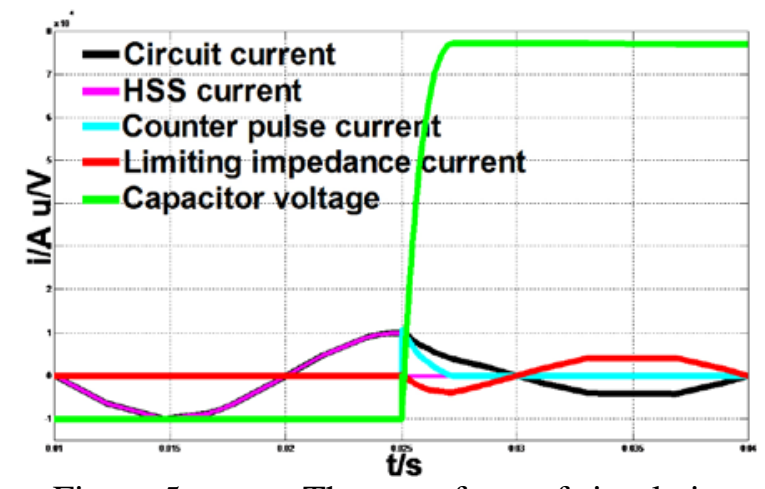

Figure $5 . \quad$ The waveform of simulation 
Table1.The results of simulation at different phase

\begin{tabular}{|l|l|l|l|}
\hline Power system phase $/\left(^{\circ}\right)$ & $\begin{array}{l}\text { First peak of } \\
\text { actual } \\
\text { /KA }\end{array}$ & $\begin{array}{l}\text { Peak current without } \\
\text { current limiters/KA }\end{array}$ & Peak current after limiting process/KA \\
\hline 0 & 2.2 & 10.13 & 5.5 \\
\hline 15 & 3.04 & 10.25 & 5.5 \\
\hline 30 & 5.2 & 10.26 & 5.5 \\
\hline 45 & 7.12 & 10.26 & 5.5 \\
\hline 60 & 9.08 & 10.26 & 5.5 \\
\hline 75 & 10.3 & 10.26 & 5.5 \\
\hline 90 & 10.89 & 10.25 & 5.5 \\
\hline 105 & 10.1 & 10.35 & 5.5 \\
\hline 120 & 8.89 & 10.26 & 5.5 \\
\hline 135 & 7.05 & 10.26 & 5.5 \\
\hline 150 & 5.21 & 10.26 & 5.5 \\
\hline 165 & 2.94 & 10.26 & \\
\hline
\end{tabular}

Owing to the uncertainty of short-circuit time, when short-circuit current occurs, phase angle is also random. Thus it is necessary to make simulation under different phase conditions. Table 1 indicates before and after the current limiting process, the simulation results of short-circuits current in different phase conditions. The simulation results show that short-circuit occurs at different phase and current limiter is accessed timely, the peak value of short-circuit current is different, and the steady state value keeps the same after current limiting process. The maximum peak value of short circuit occurs near $90^{\circ}$, the peak value of short-circuit current exceeds $10 \mathrm{kA}$.

Prototype production and experimental verification

The prototype we designed can be operated in low voltage level. The experimental voltage of prototype is $1 \mathrm{kV}$. The purpose of this experiment is to verify the theory and the correctness of simulation. Parameters of every component of current limiter prototype are: a $1 \mathrm{KV}$ high speed vacuum switch which is composed of high-speed repulsive actuator, permanent magnetic actuator[5], and vacuum arc extinguish chamber. It is verified by ansoft simulation and experiment, the time of breaking to full stroke is less than $1.5 \mathrm{~ms}$, the tree-phase actuator can be controlled and operated at the same time; VRRM of all controlled silicon is $1 \mathrm{kv}$; the maximum pulse current that is allowed to pass is $1 \mathrm{kA}$; the rated voltage of general capacitor is of $500 \mathrm{~V}$ and its rated capacity is of $110 \mu \mathrm{F}$; value of the inductance is $35 \mu \mathrm{H}$.

Short-circuit current of industrial frequency is produced through the loop which is composed of three-phase regulator and small resistance with high power. The tested waveform in current-limiting experiment is shown in figure 6. Each waveform represents the current limiting state of phase A, phase $\mathrm{B}$ and phase $\mathrm{C}$ respectively, among which, the yellow curve represents current-limiting waveform of fault current at both ends of current limiter; the blue curve is the waveform of fault current that is cut off by high speed vacuum switch under the support of counter pulse circuit.

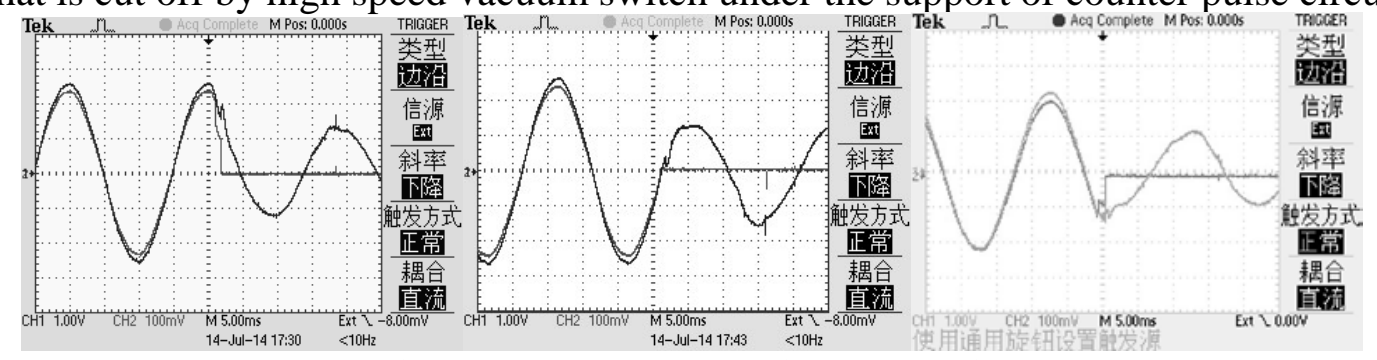

Figure 6. The experimental results

From each waveform in figure 6 , we can see that:

$1.5 \mathrm{~ms}$ after detection of the fault current, according to phase of phase A's fault current and the control strategy, the phase A, B and C input corresponding counter pulse circuit. The arc in high speed switches of phase A, B and C occur forced zero point, and the arc in each high speed switch extinguishes, the current is transferred into resistance successfully. It is clear to see from the current waveform, that every fault current is limited into about $45 \%$ of original fault current. 
From current-limiting waveform of phase B, we can see that when the high speed switch of phase B reaches full stroke position, it is just at natural zero point. Thus it is in accordance with the theory of current limiter in industrial frequency zero point. It is not necessary to input counter pulse circuit to make forced zero, the arc extinguishes at the industrial frequency zero.

\section{Conclusion}

This paper presents a fast current limiting device based on the principle of current commutation, so it quickly and effectively limits the damage to the entire system caused by the first peak of the fault current. The control strategy at any phase of the three-phase short-circuit current is presented here, the central element decides to send a relevant control signal or not according to the control strategy. Simulation and experimental analysis of this current limiting device is conducted, the results is consistent with the theory. The fault current limiting process can be completed quickly at any phase, thus it improves the stability of the system.

\section{Acknowledgment}

This work was supported in part by the National Natural Science Foundation of China (Project Number: 51277019) and in part by the National Natural Science Foundation of China (Project Number: 51337001).

\section{References}

[1] Koshida Y, Koyama K, Sasao H, et al. Development of the high speed switch and its application. In: Proceedings of the IEEE Industry Applications Conference. St. Louis, Misouri, USA: 1998. 2321 2328.

[2] Shumin Sun, Hongshun Liu, Qingmin Li, et al. Research Overview of Fault Current Limiters in Power System[J]. Power System Technology. 2008, 32 (21) 75-79.

[3] Weidong Liu. The Limiting Device Based on HSS and Current Commutation at Frequency Zero. Technical Reports. 2008.

[4] Xueliang Yang. The Study of Current Limiting Devices Based on Current Commutation at Industrial Frequency Zero:[Master Dissertation].Beijing: The Electrical Engineering Department of Tsinghua University, 2008.

[5] Enyuan Dong, Bo Li, Jiyan Zou. Experimental Performance Analysis of Ultra-high Speed Repulsion Mechanism and Permanent Magnetic Mechanism. High-Voltage Electrical Apparatus, 2007, 43(2):125 126. 